\title{
Territorialidad y sustentabilidad urbana en la Zona Metropolitana del Valle de México
}

\section{Territoriality and urban sustainability in the Metropolitan Zone of the Valley of Mexico}

\author{
Guillermo Torres-Carral*
}

\begin{abstract}
In this article we perform a theoretical-methodological approach to one of the current explanations on sustainable development, specifically urban: the so called territorial approach, taking into account the paradigmatic case of the Metropolitan zone of the Valley of Mexico (MZVM). The fundamental aim is to reflect on the analytical-cognitive interconnections that a new sort of development implies and, at the same time, analyze the practical consequences it leads to and to carry out a balance from its contribution and limitations. To do so, we resort to the general study of territorial approach; then, we considered the differences in the rural and urban spheres. Hence, only some reflections are formulated in relation to its scope in MZVM, and the difficulties that said approach carries, which here we consider insufficient to contribute to urban sustainability.
\end{abstract}

Keywords: territory, development, urbanization, planetariness.

\section{Resumen}

En este artículo se realiza un acercamiento teórico-metodológico a una de las explicaciones actuales en torno al desarrollo sustentable, específicamente urbano: el denominado enfoque territorial, tomando como referente el caso paradigmático de la Zona Metropolitana del Valle de México (zMvM). El propósito fundamental es reflexionar sobre las interconexiones analítico-cognoscitivas que implica un nuevo tipo de desarrollo y, al mismo tiempo, analizar las posibles consecuencias prácticas a que conduce; así como realizar un balance a partir de sus aportaciones y limitaciones. Para ello se estudia en general el tema del enfoque territorial; y luego se consideran las diferencias en los ámbitos rural y urbano. De esa forma, se formulan sólo algunas reflexiones en relación con su alcance en la ZMVM, y se destacan las dificultades a que conlleva dicho enfoque, que aquí se considera insuficiente para contribuir a la sustentabilidad urbana.

Palabras clave: territorio, desarrollo, urbanización, planetariedad.

\footnotetext{
* Universidad Autónoma Chapingo, México. Correo-e: gatocarr@hotmail.com.
} 


\section{Introducción}

La revisión de los estilos del desarrollo económico y social con una perspectiva socioambiental, se encuentra detrás del debate teórico-práctico respecto del desarrollo sustentable. Esta tarea resulta indispensable para atender las exigencias de la sociedad contemporánea, que consisten en la necesaria coincidencia entre las leyes de la naturaleza y los procesos socioeconómicos, en este caso sociourbanos (Cantú, 2006). Empero, ello significa sólo un punto de partida, que desde luego resulta insoslayable, para comprender los complejos problemas urbanos en el marco de la crisis de civilización (Leff, 2004).

A lo anterior hay que añadir que no basta con comprender la dinámica de la globalización per se, sino que es necesario considerarla, al menos de forma paralela, junto con un desarrollo local y, obviamente, bajo el control de la población involucrada, ya que la conjugación de los factores externos e internos ha sido siempre característica fundamental de todo desarrollo económico y social exitoso. En estas circunstancias surge el enfoque territorial del desarrollo en lo que respecta al mundo rural y al urbano, así como desde la perspectiva de la sustentabilidad. En ambos casos, es necesario conjuntar estas distintas miradas ante la necesidad de revertir la degradación socioambiental, en el campo y la ciudad, y así poder avanzar a una síntesis neocivilizatoria; enfatizando el caso de la megalópolis de la ciudad de México.

\section{El enfoque territorial del desarrollo}

\subsection{La acumulación de capital en las ciudades}

Recientemente ha cobrado gran relevancia el debate sobre el desarrollo sustentable (sobre todo rural, pero también urbano), en particular mediante el enfoque mencionado. Esto se refleja en la discusión de cómo alrededor de la noción de territorio se establece una serie de premisas y se desprenden consecuencias que, se aduce, pueden ser las más adecuadas para enfrentar el mundo en ruinas que ha engendrado el fundamentalismo neoliberal, entendido éste como la ideología contemporánea del capitalismo en el ámbito global.

De entrada, se puede afirmar que el territorio es, primero, una categoría geopolítica (y geoeconómica), independientemente de su uso en disciplinas como la ecología, geografía, economía, política o la sociología. En ese sentido, tal concepto supone el dominio de un territorio sobre otros, por lo que territorialidad implica a la vez la desterritorialidad. 
Como punto de partida hay que considerar la categoría económica que sin duda ayuda a comprender los procesos de integración territorial. Ésta es la renta de la tierra, la cual constituye la base del desarrollo espacial en el capitalismo y representa (junto al capital invertido), en última instancia, la principal determinación explicativa (como resultado del monopolio y de la propiedad del suelo) ${ }^{1}$ del territorio y también de su ordenamiento (Jung, 1972); es por tanto su base racional. Además, como instrumento analítico, la renta del suelo permite entender la relación entre la inversión realizada, la propiedad territorial y la riqueza natural del sitio explotado por el capital. De esta forma, el monto de la renta urbana determina el precio del suelo, así como la eventual inversión de capital a realizarse (Marx, 1985: 8, 792). Así, esto es lo que está detrás de la configuración de los conglomerados urbanos y su desarrollo espacial e histórico.

Lo anterior se traduce en que, en las circunstancias del modelo socioeconómico dominante, la renta urbana tiende a crecer más que la agrícola; todo ello impulsado por el modelo urbanístico en funciones y sus correlativas políticas antiagrarias y antiagrícolas (a raíz del Tratado de Libre Comercio de América del Norte, TLCAN). Su resorte principal es, por un lado, la ubicación de las inversiones de capital en sitios que no necesariamente poseen recursos naturales abundantes, sino que más bien se encuentran cerca de los centros comerciales y de las redes de servicios, sobre todo el transporte de personas y, por el otro, la enorme concentración del capital en relativamente pequeños espacios geográficos; todo lo cual demanda enorme cantidad de servicios ambientales y subsidios, por lo que, si no se respetan los ecosistemas y las comunidades ahí asentadas, la depredación se extendería sin límites como consecuencia de continuar fomentando este estilo de desarrollo urbano extensivo.

Si bien la perspectiva estudiada se acerca a la idea de la sustentabilidad, porque considera al territorio-población como un microcosmos que hay que integrar, también nos aleja de ella, ya que ignora la discontinuidad entre los procesos territoriales y, además, contribuye inevitablemente a borrar su inherente diferenciación social en aras de un pretendido desarrollo y ordenamiento territoriales. Pero, en este contexto, surgen las siguientes preguntas: ¿cómo ordenar algo que, desde sus orígenes, ha crecido en forma por demás desordenada y sin ningún tipo de planeación? Y también: ¿es absolutamente necesario reconstruir (y deconstruir) la gran ciudad insustentable para poder construir otra basada en la compatibilidad entre sus componentes? Tal es el caso de las dos grandes opciones que se le presentan a la zMVM.

${ }^{1}$ Éstas constituyen las bases (Marx, 1985) de la renta diferencial (asociada a la fertilidad natural o inducida del suelo) y la absoluta (derivada del monopolio de la propiedad territorial). 
No debemos perder de vista que la configuración urbana del espacio (Harvey, 1977; Lipietz, 1979) y el poder territorial, en los hechos son expresiones de las necesidades de la acumulación del capital (que rebasa el ámbito del territorio), el cual es móvil por naturaleza y, de hecho, construye (y reconstruye) su propio territorio de acuerdo con el comportamiento de la tasa de ganancia y, en este caso, al correspondiente modelo de acumulación para un país en desarrollo. Y también puede decirse que, en una sociedad de mercado (capitalista), los alimentos, las materias primas y la maquinaria (más aún la fuerza de trabajo) no pueden adquirirse considerando al territorio como si constituyera un mundo aislado (de ahí que la migración rompa la integración territorial a consecuencia de la exclusión y expulsión de que es objeto).

Lo anterior ayuda a entender la interrelación entre poder (económico y político) y territorio, tomando en cuenta dos formas fundamentales, las cuales no son excluyentes: 1) la extensiva (difusa), en que el territorio se expande de forma horizontal; y 2) la intensiva (compacta), donde el dominio territorial se expresa verticalmente, aunque mantiene ciertos espacios subordinados (desterritorializados) ${ }^{2}$ al territorio central. Hay que adicionar que éstas son dos formas distintas pero complementarias de la concentración del capital y de la expresión del poder territorialmente.

En el caso de la ZMVM predominó la primera forma (años sesenta y setenta del siglo $\mathrm{xx}$ ) mediante una red de anillos rodeando sus centros urbanos y, después, con el surgimiento de una ciudad con muchos centros (fines de los ochenta). Aunque la ciudad de México, en realidad, constituye una ciudad de ciudades inmersa en una expansión sin freno. De ahí la necesidad de avanzar hacia la forma intensiva mencionada.

También se puede añadir una tercera pregunta: ¿de qué sirve darle vitalidad a la ZMVM, si ello ocurre en contra de la racionalidad que supone la conservación e integridad de los ecosistemas y de la cuenca de México (y su fragmentación), así como del bienestar y convivencia de sus poblaciones?

Así, es necesario situarse en el plano de la mencionada cuenca como el espacio geográfico en que se desenvuelve la ciudad de México y su zona metropolitana. En este tenor, es fundamental distinguir entre la unidad territorial que representa la cuenca $\left(9,000 \mathrm{~km}^{2}\right)$ y la dinámica de la ZMVM, constituida como un espacio geográfico físicamente dependiente de aquélla (incluyendo su periferia de municipios conurbados), aunque dicha

${ }^{2}$ La desterritorialización se entiende como la "cara oculta, pero real, de la aparente [promoción de la territorialidad] operada por el aparato de Estado, a través de la organización impuesta a la tierra, sobrecodificándola [...] pérdida de la relación natural de la cultura con los territorios geográficos y sociales". Mientras que la reterritorialización se define como "Relocalización territorial negativa, parcial, de las viejas y nuevas producciones simbólicas” (Biagni y Roig, 2008: 171-172). 
zona urbana constituye un territorio independiente del área políticoadministrativa que representan el Distrito Federal (D.F.) y el Estado de México (Iracheta, en Ward 2004); ${ }^{3}$ y ésta a su vez con respecto al área histórica de la ciudad de México (Cantú, 2006; Moreno Pérez, 2008). Además, hay que tener en cuenta que, como plantea Graizbord (2002: 9), hay una fragmentación de los ecosistemas que son la base natural del territorio, ya que "La división político-administrativa no coincide necesariamente con la delimitación de los ecosistemas o con los rasgos naturales del terreno, o con los recursos que necesariamente forman parte de procesos cíclicos o cadenas tróficas".

\subsection{Las miradas desde el territorio}

Con la finalidad de alcanzar el objetivo de este artículo, es necesario analizar el concepto de territorio y su relación con la sustentabilidad urbana (Dempsey et al., 2009); ambos temas desde luego son complementarios. Empero, esto sólo es posible si se adopta un diálogo entre sus diversos intérpretes (desde los mundos rural y urbano). Todo ello permitirá confrontar la invasión territorial de la ZMvM con la defensa de los territorios local y comunitario, no nada más desde su periferia sino desde los mismos centros urbanos.

Por este motivo, a continuación se consideran varios ejemplos de investigadores que proponen el enfoque analizado desde la óptica de la sustentabilidad. Primero se aborda a partir de la dimensión del desarrollo rural, ya que es en ese terreno donde ha habido mayor difusión del tema y, posteriormente se aborda el tema del desarrollo urbano sustentable.

De esta manera, Schejtman y Berdegué consideran que el territorio "es una construcción social, y no un espacio 'objetivamente existente' en virtud de variables físicas o económicas" (2003: 2). En ese mismo tenor Echeverri afirma que "Es sobre este espacio que se define la idea de territorio, cuando se le incorpora su dimensión política" (2006: 202). Por otro lado, Linck señala también que representa un "espacio apropiado", y que "el territorio es un espacio con personalidad propia que ninguna ley geométrica permite entender" (2006: 254).

Es necesario destacar que estas definiciones corresponden básicamente al tema del territorio en general, aunque su principal propósito es su estudio en el análisis del desarrollo rural sustentable. Mientras que, por su parte, los intérpretes urbanos del territorio fallan (como se ve más

${ }^{3}$ Concentran más de la mitad de la población de la ZMvM, ocupan más de 50\% del área urbanizada y en ellos ocurre, a partir de los ańos ochenta, la mayor parte del crecimiento de la Zona Metropolitana de la Ciudad de México ahora denominada oficialmente del Valle de México (Iracheta, en Ward, 2004: 493). 
adelante), en general, porque excluyen las realidades del mundo rural (y de la agricultura). Resulta entonces que la primera interpretación aquí citada sobre el desarrollo territorial (es decir, la rural) es más amplia, puesto que en ella se incluyen las actividades no agropecuarias.

Un tema asociado al territorio es la competitividad. En el enfoque que nos ocupa (Schejtman y Berdegué, 2003: 4) se considera que en un territorio hay tres niveles diferentes pero complementarios y subsidiarios de competitividad: la competitividad privada de las empresas que operan en su espacio, la sistémica que ofrece el territorio en términos de potencial de productividad sumada, y la territorial, concebida en términos de rentas sociales.

Por su parte, para Echeverri y Moscardi (2005: 67) "[a] los agentes individuales les interesa maximizar sus rentas privadas, $y$ al colectivo territorial, maximizar sus rentas sociales"; sin embargo, la competitividad rebasa el ámbito territorial, porque en realidad es sistémica.

No obstante, se plantea una armonización entre el mercado y el territorio y no una contradicción entre ambos (y menos aun al interior del territorio), lo cual tiene importantes repercusiones prácticas: "El objetivo central es proporcionar instrumentos para el aprovechamiento de la economía de mercado predominantes en el capitalismo global actual, en beneficio de la mayoría de los pobladores rurales y de los países de menor desarrollo relativo del continente americano" (Echeverri y Moscardi, 2005: 69). Ello es, desde luego, un asunto (geo)estratégico; sin embargo, la desigualdad socioeconómica es inherente a la economía de mercado. Lo que faltaría entonces es avanzar hacia un modelo alternativo, ya que sólo así el mercado podría ser un medio para satisfacer las necesidades sociales; mas no como en el modelo vigente, puesto que en él constituye un fin en sí mismo.

Se puede decir que tal idea no es característica del capitalismo, ya que es el capital el que impone su propio orden económico, por lo que el territorio (como concepto y realidad concreta), y su desarrollo, se subordinan a él. En consecuencia, para que se pudiera dar dicha armonía, se requeriría la autodeterminación de la población local y también el control social del (gran) capital privado. Es posible añadir que también ha faltado destacar la trascendencia de la cooperación, y del sistema cooperativista, como el elemento fundamental para que los objetivos del desarrollo territorial se puedan cumplir.

A pesar de lo anterior y de la riqueza de un concepto integrador como el de territorialidad, se llega a concebir al territorio como una entidad que se entiende por sí misma; exagerando sus virtudes y convirtiéndola metafísicamente en un verdadero sujeto (Ramírez, 2006: 51). 


\section{El enfoque territorial urbano sustentable}

Como consecuencia del calentamiento global, así como de la necesaria transición mundial hacia un desarrollo basado en los principios de sustentabilidad y compatibilidad, son necesarias múltiples transformaciones que son esenciales para la supervivencia humana y de los ecosistemas terrestres.

El desarrollo urbano sustentable surge así como una posible alternativa de solución a la degradación socioambiental de las ciudades. Se funda en la necesidad de mantener la continuidad en los flujos de energía y de materiales, lo que contribuye a renovar los ciclos de la naturaleza, ahora mediante las actividades económicas y sociales urbanas (y rurales) favorables a aquéllos.

El desarrollo sustentable de las ciudades con un enfoque territorial ${ }^{4}$ no es un tema nuevo; sin embargo, no es fácil ofrecer una definición coherente y con un sentido de aplicabilidad práctica.

Entre algunas destacadas aportaciones, Iracheta incluye como componentes de la sustentabilidad urbana la "viabilidad económica, el desarrollo social, ordenamiento territorial, y sustentabilidad ambiental" (1997: 16). Aquí ya aparece la variable territorial paralelamente a la de sustentabilidad ambiental. Sobre lo primero, más adelante se analizan algunos de sus bemoles; acerca de lo segundo, habría que tener en cuenta que el ambiente no puede reducirse a los aspectos ecológicos.

Igualmente se puede mencionar la ley de Desarrollo Urbano Sustentable del estado de Puebla (2003), donde éste se define como "Establecer las normas para planear, ordenar y regular la fundación, conservación, mejoramiento y crecimiento ordenado de los centros urbanos de poblaciones, así como de las zonas y conjuntos urbanos".

Con estos ejemplos, la idea del ordenamiento territorial se muestra fundamental para avanzar en la dirección de un desarrollo urbano sustentable, lo cual aparentemente es suficiente; pero si no se ordena la población, y más aún el capital (mediante su control y vigilancia por parte de la sociedad), tales esfuerzos serían insuficientes para lograr los propósitos de una transición ambiental.

Por su parte, para Barton el desarrollo urbano sustentable "supone fortalecer la legislación e incorporar las variables ambientales y de integración social en los instrumentos de planificación territorial' (2006: 36). Así, nos acercamos a la noción de territorio como eje del desarrollo urba-

\footnotetext{
${ }^{4}$ Es necesario destacar que territorio no es lo mismo que enfoque territorial, pues aquél puede verse desde otras perspectivas.
} 
no, sin establecer las mediaciones que lleven a definir sus límites (físicos), fronteras (políticas) y contraposiciones (socioeconómicas) fundamentales.

Paralelamente, Borja y Castells afirman que "La organización del espacio urbano y de las actividades que se realizan en el seno de una ciudad debe tener condiciones adecuadas a la habitabilidad de sus habitantes. El mantenimiento de estas condiciones debe incorporar la dimensión temporal -asegurar las condiciones de habitabilidad a generaciones futurascomo la dimensión espacial, sin comprometer la sostenibilidad de sus diversos ámbitos de influencia, desde las áreas próximas hasta aspectos globales" (1997: 196).

Para estos autores, si bien el aspecto espacial es fundamental, éste se encuentra al menos al mismo nivel que el aspecto temporal. Por otra parte, la idea de sostenibilidad es, por lo demás, ambigua ya que se limita a garantizar la durabilidad de la realidad capitalista en el tiempo, aunque aún sin incorporar las condiciones ecológicas del desarrollo (Leff, 2004), como es en el caso del significado del término sustentabilidad; sin embargo, ambas palabras tienen la misma raíz, lo que implica que las dos (al igual que el término anglosajón sustainable del cual son traducción) tienen la gran limitación, aparte de su ambivalencia, de ser condescendientes generalmente con los mecanismos de la acumulación de capital.

Por último, los llamados principios del movimiento Smart Growth plantean: "Queremos ciudades bellas, amables, equitativas. Esto supone una política urbana moderna, con sentido de territorio. Supone fortalecer la legislación e incorporar las variables ambientales y de integración social en los instrumentos de planificación territorial' (Mc Donough y Braungart, 2002: 2).

Para otros autores, y en el marco del paradigma de la agricultura urbana, es necesario construir ciudades verdes, con suficientes empleos y alimentos (Schenna, 1995: 4). En este caso, al introducir la variable alimentos, se incluye un aspecto fundamental frente a la crisis alimentaria global actual y el papel que tienen las ciudades para producir su propio valor calórico, sus alimentos (lo que presupone generar suficiente biomasa para combatir los efectos del cambio climático mediante la captura de carbono) y reducir sus graves efectos ecosociales.

No obstante las anteriores aportaciones, como mencionamos, éstas tienen la limitante de contemplar al mundo urbano separado del rural y de ignorar la trascendencia histórica y cultural de la agricultura.

Se puede agregar que otros investigadores ponen el énfasis en el papel de las políticas públicas y la participación ciudadana, que es un aspecto fundamental de la territorialidad y la sustentabilidad. Así, para Kasperson la sustentabilidad urbana sólo es posible mediante "políticas ambientales, esfuerzos coordinados de organizaciones no gubernamentales, tecnologías 
innovadoras y una ciudadanía con una aguda capacidad de recuperación" (en Ezcurra et al., 2006: 8).

No obstante sus ventajas, el enfoque territorial urbano no está exento de desventajas argumentativas y prácticas, la principal radica en que los problemas socioambientales sólo en parte son locales y territoriales.

Asimismo es necesario distinguir entre territorialidad y tierra, lo que implica abordar sus diferencias cualitativas y no sólo cuantitativas (por ejemplo, el territorio es más amplio que las áreas agrícolas, porque abarca el territorio simbólico, pero menos amplio que la tierra). Por ello, el territorio está integrado a otros conceptos más generales (planeta-tierra, globo, sociedad, etcétera).

Por último, y en el ámbito del enfoque territorial urbano, se puede agregar que las economías urbanas también aportan servicios ambientales (industrias de separación de basuras, reciclaje, tratamiento de aguas, entre otros).

\section{Limitaciones y aportaciones del enfoque territorial}

A pesar de su indudable influencia política, este enfoque genera muchas interrogantes e incluye evidentes limitaciones, aunque también claras aportaciones en el contexto del análisis teórico-metodológico y de su probable contribución a la solución de los problemas socioambientales (en especial en la ZMVM). Veamos, entre las primeras:

1. Todo desarrollo socioeconómico no distorsionado, y más o menos armónico, se basa en el desarrollo local (y el fomento del mercado interno), pero también en su integración al mercado mundial. Esto es tan cierto dado que la ciudad de México es cada vez más global (García Canclini, 2003). Sin embargo, poco se ha avanzado en el desarrollo territorial de la gran ciudad de México.

2. El territorio es un producto del desarrollo del capital cuya lógica fácilmente se amolda al espacio construido y al enfoque aludido para maximizar así sus ganancias (economías de escala y de aglomeración), integrando para este fin a las distintas actividades económicas ( $\mathrm{y}$ en segundo plano ecológicas, pues en el territorio la ecología se rinde ante la economía, con el modelo actual).

3. El espacio carece de una sustentabilidad territorial per se, porque está involucrado en una centralización económica, política, cultural, etcétera.

4. El enfoque resulta incapaz de modificar el diseño y aplicación de las políticas públicas (incluso territoriales), ya que provienen desde los centros del poder, no del territorio. 
Mientras que entre los aportes del desarrollo territorial, específicamente urbano, podrían citarse los siguientes:

1. Genera acciones para lograr una economía del espacio urbano, con sus efectos positivos en mayor eficiencia energética y mejoramiento social; así como de ahorro de recursos naturales y humanos empleados (a diferencia de la ZMVM donde prevalece el despilfarro).

2. Fomenta la integración técnica de las actividades económicas entre sí (y con las actividades ecológicas), lo que contrasta con la configuración socioproductiva actual de la ciudad.

3. Implica aprovechar y distribuir la fuerza de trabajo local y su adecuada retribución; lo que choca con las externalidades negativas (pérdidas).

4. Sugiere la posibilidad de inducir inversiones mediante el ahorro financiero local.

5. Permite avanzar hacia una mucha mayor participación de la población urbano-rural en la solución de sus problemas socioambientales.

6. Supone la reapropiación social, aunque de manera circunscrita a las fronteras geopolíticas establecidas institucionalmente.

Las contribuciones de este enfoque se pueden resumir cuando Echeverri y Moscardi afirman que:

El territorio no sólo es el escenario espacial [...] sino que también involucra la dimensión espacial delimitable de la unidad geosocial que garantiza la continuidad transgeneracional y el arraigo ancestral de los grupos humanos que permanecen en dichos territorios, en la cual la relación entre sociedad y territorio está mediada, inevitablemente, por las relaciones de producción, distribución y consumo (olvida la circulación) que caracterizan a los modos de producción construidos sobre éste y otros territorios con los cuales se vincula (2005: 58).

Aunque hoy, frente a fenómenos como el migratorio (entre otros temas) es tan amplio y complejo que la noción misma de territorio no lo puede abarcar.

\section{El enfoque territorial: hacia un balance}

Aunque el enfoque territorial urbano (y rural) sustentable es parte destacada de las políticas oficiales, ${ }^{5}$ su fundamento se encuentra en la realidad ecosocial que lo produce como concepto y práctica política (tanto gubernamental como no gubernamental), e igualmente en la necesidad de

\footnotetext{
${ }^{5}$ Un ejemplo del enfoque territorial aplicado se encuentra en la Ley del Desarrollo Rural Sustentable (2001).
} 
supeditar el ciclo económico al ecológico; modificando el tipo de intervención humana en la naturaleza. Sin embargo, dicho enfoque genera muchas interrogantes. Veamos por qué.

1. La ambigüedad del territorio radica en que el mismo término puede ser empleado como territorio local, regional, nacional, internacional, etcétera.

2. La territorialidad es sólo una de las tantas visiones que están presentes en el campo y la ciudad; pero debe complementarse con las otras.

3. Se mencionaron las ventajas y desventajas del enfoque, destacando que su trascendencia se circunscribe a constituir sólo parte de un proceso interactivo entre el campo, la ciudad y los ecosistemas. De ahí que el territorio no elimine sus contraposiciones. Entonces debe ampliarse incorporando las políticas públicas al desarrollo sustentable. De esta forma, el enfoque analizado representa una corriente específica de la sustentabilidad, la cual está implicada en el reconocimiento crítico de la enorme concentración del poder económico y político que, sin embargo, no se cuestiona explícitamente. Pero sin la descentralización de éstos, la idea del desarrollo territorial no tendría éxito.

4. Sin embargo, en el caso de la ciudad de México y su zona metropolitana, el análisis precedente corrobora la importancia de la territorialidad para el desarrollo urbano sustentable. Esto resulta inevitable frente a la sumisión de los territorios (conurbados) que pierden su autonomía socioambiental, además de que la mancha urbana acaba con las reservas ecológicas y culturales (zonas rurales).

5. Por lo anterior, un punto de vista que no distingue diferencias entre el campo y la ciudad, y las actividades agropecuarias y no agropecuarias, pareciera muy apropiado y conveniente para un megadesarrollo urbano que acaba con todo y al que muy frecuentemente se le llama desarrollo sustentable, y que se reduciría a la llamada sustentabilidad territorial.

\section{Territorialidad, multisectorialidad, transversalidad y planetariedad}

Para arribar a una visión compleja del territorio es necesario incluir al menos los siguientes conceptos, los cuales corresponden a dimensiones complementarias que son indispensables para su comprensión y discusión.

Primero, la territorialidad es una concepción que destaca la relación entre el espacio físico y la población de un lugar determinado. Ésta de- 
nota mayor amplitud que la suma de los usos actuales del suelo, pues además de incluir reservas territoriales, incorpora espacios no productivos; todo ello dentro de un proceso de apropiación social y local.

En segundo lugar, hay que señalar que uno de los grandes límites del territorio, que constituye su propia frontera y que lo rebasa continuamente, es el concepto de multisectorialidad. Ésta trasciende al territorio (y por tanto al capital inmobiliario) y supone el funcionamiento de la movilidad del capital por medio de la industria, la agricultura, el comercio, la banca y los servicios (incluido el rol del consumismo en la sociedad actual, que nada tiene que ver con el territorio); y desde luego sin ignorar la influencia de las acciones gubernamentales.

La multisectorialidad acerca a la idea de integrar las actividades económicas existentes, pero dentro y también fuera de un territorio específico; mientras que la territorialidad implica la realización de acciones sólo en un espacio bien delimitado; igualmente, puede añadirse la intersectorialidad como parte de la misma integración.

Tal concepto hace énfasis en que los procesos urbanos y rurales tienen dos dimensiones: una estructural y otra histórica; en una tomamos en cuenta el cambio a lo largo del tiempo dentro de un mismo espacio, y en la otra, en el mismo tiempo se encuentra la sincronía entre los espacios diversos; y es ahí donde destaca.

En la multisectorialidad no sólo se manifiesta el entramado urbanorural en un área determinada, sino también en el sentido global. Esto implica que atender a todos los sectores socioeconómicos significa entender sus vínculos, así como sus relaciones de dependencia; en donde evidentemente una esfera domina a la otra, esto es: el territorio está atravesado por el poder económico y político.

En tercer lugar, es necesario agregar la transversalidad como un elemento adicional sin el cual es imposible la sustentabilidad urbana en el marco del desarrollo territorial del ecosistema urbano (Huang y Shu, 2010).

La transversalidad (Tudela, 2008) consiste en un proceso de integración de las distintas instancias gubernamentales, a los fines, en este caso, del desarrollo sustentable; mas aquélla no debe quedarse ahí, pues debe incidir en el corazón mismo de la sociedad civil.

Finalmente, la planetariedad (Morin y Kern, 1993) permite ubicar la territorialidad sólo como parte del mundo entendido como el ecosistema planetario y abarcando todos los ecosistemas y ambientes terrestres. Sin ella se caería en la trampa de la fragmentación. 


\section{El territorio y la reapropiación social}

Un resultado al que puede llevar el balance del enfoque estudiado en este artículo es que, a diferencia del comportamiento de la producción y el mercado capitalistas, que están dentro de la lógica de la continuidad técnica y de la valorización del capital, la territorialidad está inmersa en la discontinuidad espacial que choca con la continuidad temporal (impregnada de la búsqueda del lucro, no de la vocación del suelo), pues la distinta combinación de factores de la producción y de diferentes formas de producción varían en los distintos territorios (que por naturaleza son heterogéneos), y esto hace imposible el ordenamiento territorial, a menos que se hagan los ajustes al modelo económico, traduciéndose en una política ambiental con mejores efectos prácticos, tanto en el campo como en la ciudad.

Así, el territorio está determinado desde fuera de él. Y en la sociedad actual depende del capital, el cual lo moldea de acuerdo con sus necesidades de acumulación.

En el caso de las ciudades, no sólo se trata de ordenar el futuro sino de reordenar el presente lleno de desigualdades sociales, y frente al necesario proceso de democratización de la economía que es una vieja demanda de los pueblos del mundo. Pero esto requiere superar el modelo de desarrollo urbano-rural desequilibrado y distorsionador en marcha.

Asimismo, hay que agregar la presencia de un grave riesgo para los pobladores, específicamente de la ZMVM, ya que la megalópolis "puede constituir el preludio de una gran catástrofe ecológica" (Ezcurra et al., 2006: 26). De manera institucional, la solución a su fragilidad y vulnerabilidad ambientales concomitantes debe incluir un pesimismo movilizador de la sociedad civil, y de las mismas conciencias de las grandes ciudades (Rees y Wackernagel 1996: 4), sobre el poco tiempo que queda frente a la realidad del cambio climático global para sostener el modo de vida actual, que ya que es insostenible.

Es por eso que, ante ese escenario, la participación ciudadana (Graizbord, 2002) surge como eje de la transformación del entorno urbano hacia otro cualitativamente diferente. Resulta esencial en la transición hacia ese nuevo ambiente y al necesario reordenamiento territorial y poblacional, así como intersectorial.

Como consecuencia, en el contexto neorrural y neourbano, los movimientos sociales corresponden a distintas miradas fragmentarias de la realidad del campo y la ciudad, pero que se pueden considerar complementarias en el marco de la reapropiación social de la vida.

Así, es posible mencionar los siguientes tipos de movimientos sociales en el campo: a) en defensa de los migrantes o para generar proyectos 
productivos; $b$ ) de lucha y defensa de la tierra; $c$ ) por la defensa del territorio (local, regional); d) movimientos ambientalistas en defensa de los recursos naturales a nivel macro; y $e$ ) movimientos por más apoyos (de todo tipo) para el campo.

Y en el caso de los procesos urbanos: a) movimientos por la vivienda; b) por la regularización de la tierra; $c$ ) en demanda de servicios urbanos para frenar la depredación inmobiliaria; $d$ ) movimientos por la democracia y los derechos humanos; y e) por la soberanía y rescate de los recursos naturales.

Por último, la defensa de los territorios agrícolas y rurales es una de las grandes lecciones que se encuentran en la noción del territorio, es donde éste es producto de la disputa por hacer prevalecer determinadas voces y miradas (que surgen de la ciudad o del campo), todo lo cual deriva en determinado tipo de acciones que puedan favorecer a sus poblaciones o bien que las induzcan a una espiral de confrontaciones en la lucha por el poder territorial (y es a lo que conduce la expansión de maquiladoras contaminantes o de los clusters).

Como se puede deducir de lo anterior, este enfoque implica abordar sólo de forma limitada algunos de estos movimientos. Sin embargo, ello no lo demerita.

\section{Territorialidad y la ZMVM}

No es agua ni arena la orilla del mar
José Gorostiza

\subsection{El territorio y las fronteras de la ZMVM}

El espacio territorial se expresa en un proceso (de expansión y contracción) continuo de construcción social. De esa forma, el territorio está delimitado por sus fronteras (que son límites convencionales), las que entrelaza con otros territorios. Y lo anterior tiene una connotación descriptiva de cómo ocurren los hechos exclusivamente en el terreno de la división político-administrativa, jurisdiccional (municipal). Pero, en esencia, constituye una determinación geopolítica, lo que implica la presencia de las relaciones de poder y de las desigualdades intra y entre regiones.

Además, no hay que ignorar que el territorio es un concepto que expresa el hecho de que determinados recursos naturales, humanos e institucionales se hallan sujetos al servicio del poder político y económico. Tal es el caso de la ZMvM.

Los procesos de expansión de la ciudad de México se han sostenido con base en el sometimiento de los ecosistemas y territorios a su alrede- 
dor, y la concomitante centralización económico-política, expresada en la subyugación de las comunidades tanto locales como periféricas. Ello define el tipo de urbanización expansiva. Y ésta, en última instancia, está determinada por la interacción con el estado de los ecosistemas naturales (y humano-social) a su alrededor, los cuales han sido modificados drásticamente por la intervención humana, sobre todo en la actualidad debido a la expansión del ecosistema urbano. Sin embargo, la ZMVM es parte integrante de la Cuenca de México, la cual, como unidad territorial de estudio, es resultado de miles de años de evolución geológica y biológica; así como producto -más recientemente- de una continua devastación provocada por el desarrollo urbano, a medida que la ciudad de México vivió momentos decisivos desde la conquista, y luego la Colonia, Independencia, Reforma, Revolución y, sobre todo, en el México contemporáneo. En todo este proceso, "Durante su conversión de plaza a suburbio y a megaciudad, la ciudad de México se ha vuelto más diferenciada y segregada" (Ward, 1991: 13); de ello nace la dicotomía urbana que le es inherente.

Por eso la megalópolis (Gottman, 1961; Harvey, 2009) de la ciudad de México, como centro del poder político nacional, se sustantiva como una frontera que se excede a si misma, lo que le permite extender su poder y territorio, pero también explicar una de las poderosas razones que ha presionado para generar una creciente "huella ecológica" (Kasperson, en Ezcurra et al., 2006: 17; Rees y Wackernagel, 1996), que no es más que un eufemismo para calcular el efecto (en este caso urbano) en términos de materiales y energía (traducibles en cantidades de tierra) generados por el consumo humano; y que es consecuencia de un hacinamiento demográfico provocado por el asentamiento de unos 20 millones de habitantes en la ZMVM -que representa sólo alrededor de $0.26 \%$ del territorio nacional (Moreno Pérez, 2008), mientras que el D.F., el 0.76\%, 1,400 km² (de los cuales $59 \%$ son suelos de conservación) que albergan a nueve millones de habitantes, más cuatro millones de población flotante-; ${ }^{6}$ además de que el D.F., la entidad político-administrativa más dinámica de todo el país (aunque cada vez más rebasada por el Estado de México), representa $21.52 \%$ del PIB nacional (INEGI, 2006), genera 23,000 toneladas diarias de basura (De la Fuente, 2006: 18) y en el año 2006 consumió la tercera parte del petróleo y electricidad nacionales y $70 \%$ del agua del acuífero de la Cuenca. Se puede agregar que, sólo en el D.F., en el año 1993 se

${ }^{6}$ Ezcurra et al. (2006: 45) proporcionan el dato de 0.48\% para la Cuenca, pero es erróneo ya que sólo el Distrito Federal tiene una extensión de $1,400 \mathrm{~km}^{2}$, es decir, $0.76 \%$ del territorio nacional (De la Fuente, 2006: 46). Por su parte, Peter Ward estimó el área urbana en $1,250 \mathrm{~km}^{2}$ para el año 1980 (1990: 72) y en 1,460 $\mathrm{km}^{2}$ para 2000 (2004: 120), la cual alcanza una densidad de 116 habitantes por hectárea. 
concentraba $56 \%$ de las empresas más grandes de todo el país (Pérez Corona, 2008); por su parte, la ZMCM representó $31.3 \%$ del PIB nacional (Iracheta, 2004: 521).

Además, es necesario destacar que diferentes tipos de ecosistemas (forestal, pastizales y una diversidad de espacios de transición) y zonas ambientales (Ezcurra et al., 2006: 35), así como diferentes culturas, siempre han estado en juego en la evolución sociocultural de la ciudad de México.

De esa manera, el resultado ha sido la ampliación del área urbana (Ward, 2004) que se reducía, de ser una mera fracción de la entidad federativa (D.F.), a la configuración de la ZMvм abarcando sus 16 delegaciones, alrededor de 59 municipios del Estado de México y uno del estado de Hidalgo; y para alimentarla en su sed insaciable, se necesita traer agua de las cuencas de los ríos Lerma-Santiago y el Sistema Cutzamala (y se ha previsto incorporar muy pronto la cuencas de los ríos Amacuzac y Temascaltepec). Por su parte, la Cuenca de México ${ }^{7}$ abarca 86 municipios y delegaciones del D.F. y de los estados de México, Hidalgo, Puebla y Tlaxcala (Ezcurra et al., 2006: 89).

Como resultado "Podría argumentarse que, actualmente, toda el área metropolitana constituye un elemento externo negativo, debido a la manera en que afectan a todos sus habitantes los problemas relacionados con las condiciones de vida que incluyen un alto nivel de contaminación atmosférica, la falta de servicios adecuados para muchos, escasez de áreas verdes, largos trayectos a los sitios de trabajo, etcétera" (Ezcurra et al., 2006: 90).

El crecimiento de la mancha urbana en el D.F. (De la Fuente, 2006: 54) ha ocasionado que el área verde per cápita sea, en cualquier caso, inferior $\left(5.3 \mathrm{~m}^{2}\right)$ a los estándares internacionales recomendados $\left(9 \mathrm{~m}^{2}\right)$; asimismo, que el ritmo anual de reducción de las zonas agrícolas sea de $7.4 \%$ y de las áreas verdes de $3.7 \%$. Ello ocurre en el contexto de la urbanización de la pobreza (más de las dos terceras partes de la pobreza en México se concentran en las ciudades, es decir, 37.5 millones aproximadamente); de la cual corresponde $60.4 \%$ al sector rural del D.F.; $76.6 \%$ en el Estado de México y 79\% en el de Hidalgo (Hernández, 2002: 17), por lo que la pobreza urbana representa alrededor de $40 \%$ en el D.F.; $24 \%$ en el Estado de México y $21 \%$ en Hidalgo.

Por otro lado, si bien el ritmo de crecimiento de la población del D.F. (Ezcurra et al., 2006: 90) se ha reducido de 0.32 a $0.24 \%$ entre los años

\footnotetext{
${ }^{7}$ Ésta se halla delimitada por la Sierra de la Cruces (poniente), Sierra Nevada (suroriente), al norte la zona volcánica de Pachuca y al sur la Sierra de Chichinautzin.
} 
2000 y 2005, el número absoluto de habitantes en la ZMvM se sitúa en 20 millones y sigue aumentando.

Se puede agregar que una característica central del desarrollo urbano efectuado es la transición del D.F., en tanto ciudad centrada en la actividad industrial, a una donde prevalecen las actividades de servicios (Ward, 1991: 143; Garza, 2003), y que en el año 2000 aglutinaba 72\% de la población empleada y mantenía el predominio económico sobre el resto del país, concentrando $21 \%$ de la fuerza de trabajo industrial en todo el país (Nuevo León 8\% y Jalisco 6\%) (Pérez Corona, 2008: 17).

\subsection{Tipo de urbanización}

La caracterización del tipo de urbanización que permite identificar la clave y el rumbo de los procesos socioeconómicos y demográficos presentes en la ciudad de México y áreas conurbadas, es un punto de partida indispensable para definir el modelo de ciudad que requieren sus habitantes, así como las posibilidades en el cumplimiento de sus derechos individuales y constitucionales (Ferrajoli, 1995).

En un principio, los procesos de metropolización conformaron un área que incluyó un centro y tres anillos metropolitanos, donde el crecimiento y la distribución poblacional mostraron marcadas diferencias (Ezcurra et al., 2006: 78, 90). Empero, recientemente el centro se ha despoblado, mas no así los anillos donde se intercala la pobreza en distintos grados. Estos procesos describen un modelo caracterizado por la extrema desigualdad social, en un contexto de una urbanización que no ha respetado nada.

Peter Ward explica que "El área metropolitana puede dividirse en una serie de tres 'anillos', que rodean el núcleo central de la ciudad; el primer anillo comprende las delegaciones y municipios inmediatamente adyacentes al Distrito Federal (49\% de la población total); el segundo incluye las delegaciones más alejadas y los municipios menos adyacentes del Estado de México (32\%); y el tercero comprende, en esencia, los municipios más alejados, los cuales están creciendo rápidamente (7\%)" (2004: 79).

Además, se avizora que el futuro inmediato del desarrollo territorial de la megalópolis (Aguilar, 2003) incluya varios centros urbanos con un núcleo central y sus anillos, en irremediable expansión hacia los cuatro puntos cardinales. Sin embargo, la delimitación de la periferia rural debe entenderse en función de toda el área urbana (zMvM) y no de dichos centros, ya que en y entre estos últimos, se está ante la continuidad urbana-urbana; tal es el caso del norte metropolitano y las amenazas de que ello se extienda al sur $\mathrm{y}$ poniente. 
Asimismo se trata, en este caso, de una urbanización invasora por su carácter extensivo que se beneficia de la pobreza y abaratamiento de los recursos naturales, lo que aunado a los subsidios en los precios de los servicios básicos citadinos, constituye un poderoso imán para la población proveniente de la periferia urbana de la ZMVM (norte y oriente) y más allá de la Cuenca de México.

En la perspectiva del nuevo tipo de urbanización de la región-ciudad de México, policéntrico y difuso (Aguilar, 2003), el proceso que revela un carácter expansivo territorialmente y dicotómico en su forma de organización (por ejemplo, Los Reyes la Paz frente a Santa Fe), produce cierto tipo de refeudalización (donde además las bandas urbanas por medio de los grafitis marcan sus territorios siguiendo la ley de la selva, o mejor dicho, la ley del revólver, Bookchim, 1978). Frente a ello la alternativa que puede ofrecerse es la transición a una ciudad compacta.

\title{
7.3. Los efectos socioambientales del modelo de urbanización de la ZMVM
}

En las condiciones actuales de los procesos de metropolización presentes en la ciudad-región, y en relación con el aprovechamiento de los recursos naturales a su disposición, hay que señalar que

\begin{abstract}
Aunque el crecimiento de la población está disminuyendo, y muchos jóvenes de clase media están emigrando a otras ciudades en busca de una mejor calidad de vida, la migración de campesinos provenientes de las regiones rurales empobrecidas hacia la megalópolis continúa, y la ciudad se sigue extendiendo sobre bosques y campos. Además, el consumo de combustibles fósiles, la cantidad de automóviles, la sustitución de bosques por áreas urbanas, y el bombeo de agua subterránea de un acuífero críticamente disminuido siguen aumentando a una tasa frecuentemente más alta que el propio crecimiento poblacional (Ezcurra $e t$ al., 2006: 241-242).
\end{abstract}

Así, el resultado es que la Cuenca abastece cada vez menos agua potable para la población de la gran urbe, aumentando aceleradamente la demanda ficticia de ella (fugas, derroche, crecimiento urbano), mientras que su capacidad máxima que sólo es para 8.5 millones de personas, se alcanzó en el año 1964.

La megalópolis de la ciudad de México ${ }^{8}$ no se podría entender si su viabilidad no se enmarca en el manejo ecosocial (y en el estudio) del territorio de la Cuenca de México, y su necesaria restauración. Éste sería sin duda el principal resultado a que han llegado las investigaciones en

\footnotetext{
${ }^{8}$ Ésta amplía el conglomerado urbano, de los límites actuales de la zMvм, hacia la integración con las metrópolis de Toluca, Pachuca, Cuernavaca, Puebla y Tlaxcala.
} 
este tema que, por lo demás, viene a confirmar que la destrucción de la naturaleza es directamente proporcional a un modelo insustentable de manejo de recursos naturales. Así, por ejemplo, Iracheta sostiene que

El problema de esto es que en lo concreto, es decir en la planeación territorial, en la distribución de los recursos públicos, en los acuerdos políticos e inclusive en la apreciación social y cultural, no existe la Zona Metropolitana del Valle de México, y por ello carece de reconocimiento jurídico y estatus político. Tanto el gobierno del Distrito Federal como el del Estado de México han elaborado sus planes y programas de desarrollo urbano y de otras materias al margen de la opinión del otro, salvo excepciones notables, como las vinculadas al ambiente, por citar un ejemplo (Iracheta, en Ward, 2004: 601).

En este contexto, los efectos ecosociales que caracterizan a la ZMVM son los siguientes:

1. La crisis hídrica: inundaciones/sequías, sobreexplotación de acuíferos, desperdicio del agua de lluvia (y su mezcla con el drenaje), contaminación de los ríos y cuerpos superficiales; muy reducido tratamiento de aguas residuales, etc. El problema de fondo es que la recarga es menor que la extracción de agua de la Cuenca (estrés hídrico). Además del costo elevadísimo que representa bombear el líquido, primero para subir el agua a la olla de la ciudad de México, y segundo, para desalojarla a través del drenaje profundo considerando la pérdida de la pendiente, derivada de los hundimientos del suelo; producto a su vez de la sobreexplotación del agua en pozos profundos. Por tanto, la crisis del agua no es un simple efecto de la disociación entre la oferta y demanda del líquido sino, principalmente, resultado de su mal manejo.

2. El continuo cambio de uso del suelo de forestal o áreas agropecuarias a urbanas, se traduce en que la cantidad de áreas verdes per cápita es insuficiente (según los estándares internacionales), mientras que las tasas de disminución de la superficie cubierta de vegetación o rural son elevadas.

3. La contaminación atmosférica, si bien ha disminuido en cuanto a algunos contaminantes (imecas), ha aumentado en relación con otros (micropartículas suspendidas), acelerando la adquisición de enfermedades por parte de la población; e igualmente ha provocado las islas y golpes de calor cada vez más intensos. Esta situación desde luego es resultado, en gran media, del voraz consumo de energía derivado del consumismo implantado como estilo de vida citadino; "La contaminación atmosférica ocurre debido a las frecuentes inversiones térmicas, al gran número y amplia distribución 
de las fuentes de contaminación y a las complejidades de los flujos regionales del aire dentro del valle central" (Ward, 1991: 93).

4. Las pérdidas económicas (y en salud) por el transporte son cuantiosas debido al tiempo que se requiere para trasladarse del hogar a los centros de trabajo y viceversa; y la cantidad de vehículos automotores ronda los 4.5 millones en la ZMVM, nada menos.

5. También es posible que la desindustrialización de la ciudad, a raíz del TLCAN, haya propiciado que se configure una ciudad global (García Canclini, 2003) moldeada por los servicios (Garza, 2003; Pérez Corona, 2008).

6. Por su parte, la economía informal, en los distintos centros urbanos de la megalópolis, se ha convertido en la opción para millones de citadinos que no encuentran otra forma de sobrevivir; representando $30 \%$ del empleo en todo el país.

7. Ante la falta estructural de ingresos y los bajos salarios, se han creado condiciones, junto a la corrupción e impunidad del poder, para provocar una verdadera espiral de violencia e inseguridad.

8. Asimismo, el problema de los residuos sólidos es cada vez más grave debido a las carencias en materia de infraestructura y capacitación tanto de la población como de los trabajadores del sector que realizan labores de recolección, separación, transporte y manejo de la basura.

9. En las partes más pobres (áreas conurbadas principalmente) de la ZMVM sigue presente la falta o graves deficiencias en infraestructura básica (agua potable, drenaje, pavimentación).

10. Las redes de la burocracia, y su corrupción correspondiente, son graves, así como su ineficacia e ineficiencia; esto se relaciona con cómo se ejerce el poder político en México, no obstante los avances en los procesos de democratización y de ciudadanización que son cada vez más fuertes, aunque todavía insuficientes y en ocasiones frenados.

11. La ciudad, además, vive presa de la falta de cortesía y amabilidad entre sus habitantes. La guerra urbana es un asunto de todos los días. Esto es producto de la incomunicación citadina, agudizada por las políticas y el modelo socioeconómico en funciones.

12. Finalmente, el denominado riesgo total está presente como una amenaza (un Hiroshima ecológico, Ward, 1991) que se cierne sobre la ciudad; igualmente, la fragilidad ante los desastres naturales, y la vulnerabilidad ante el cambio climático, la están convirtiendo en una ciudad-desierto. Pero más que un riesgo total y una gran catástrofe, la ciudad misma se significa como una serie de catástrofes permanentes (Davis, 2003). 


\subsection{Las respuestas al modelo de urbanización: la sustentabilidad urbana}

No hay que caer en el error de pronosticar un mayor deterioro socioambiental sin señalar cuáles serían las características de un modelo alternativo (que no puede limitarse a la descentralización y racionalización de subsidios), con el cual se articularían las políticas ambientales y económicas. Éste tiene que ver con resolver la disyunción entre la megaciudad y la posibilidad de construir una red de ciudades verdes; así como el de la superación o fortalecimiento de una ciudad basada en la dicotomía urbana. En este sentido, se manifiesta que la gran ciudad está partida en dos grandes segmentos que se yuxtaponen, y donde la continuidad espacial está intercalada por dinámicas opuestas: de esta forma, existen zonas de muy alto poder adquisitivo que en ocasiones se separan, pero más bien acompañan a los multitudinarios sectores populares.

Por otro lado, no hay que darles más importancia a las políticas gubernamentales que a la expansión y consolidación de las redes ambientales para la solución de la problemática estudiada, la cual no se restringe a la lucha contra la contaminación; mientras que no se le atribuye suficiente trascendencia a la organización y movilización social (que fue fundamental como reacción tras el terremoto de 1985). Por tanto, lo anterior tiene que ver con la puesta en práctica de una democracia participativa.

Puede señalarse que, aun con el enfoque territorial, no basta con la necesidad de impulsar la descentralización pues, sin el desarrollo rural, no podrían entenderse los patrones actuales de hiperurbanización (Castells, 1983) en América Latina. Por ello no puede desdeñarse el fomento de la agricultura y otras actividades rurales (dentro y fuera de las ciudades).

La migración a la ZMVM no sólo se explica por los subsidios que ofrece, sino también por la presencia de un modelo rural caracterizado por la exclusión y el abandono. De manera que un adecuado desarrollo rural en el aspecto territorial, pero también multisectorial, ayudaría más que la eliminación de tales subsidios urbanos, a un reordenamiento de la población, desde luego junto con el territorial. Pero lo más importante no es este último, sino el reordenamiento económico y político. En pocas palabras, se trata del nivel y estructura de la gobernabilidad ambiental, máxime frente a la bomba de tiempo que representa la zona urbana.

El experimento de la expansión metropolitana trae sus secuelas en relación con la racionalidad económica y ecológica: por tanto de la misma economía urbana. Esto es así porque las erogaciones sin compensación que hace el gobierno es un arma de doble filo.

Lo anterior se traduce en que al no aumentar los precios reales de los servicios y mantener los costos político-administrativos de la gran ciudad, 
ello genera una ventaja injusta que se convierte en un polo de atracción de la población proveniente de lugares cada vez más lejanos (lo que contrasta con las desventajas de precios más altos en sus comunidades de origen); no obstante, dichos subsidios se otorgan para contrarrestar el atraso estructural de las sociedades urbanas en los países en desarrollo, aunque generan desde luego efectos perniciosos al reproducir la desigualdad urbano-rural.

Esto es así porque además de ser recomendaciones que benefician al poder, en realidad no ayudan a resolver los problemas que se sitúan en la esfera de las relaciones sociales de producción; no en la continuidad/ discontinuidad territorial.

Otro problema crucial asociado es que la pérdida de competitividad de la ciudad se hace cada vez más evidente ante la magnitud de los mencionados subsidios, los cuales tienen un doble carácter, ya que son un mal necesario; esto es, implican una serie de ventajas, pero éstas son artificiales y significan a la vez limitaciones fiscales para el gobierno de la ciudad.

Como resultado del atractivo citadino, sigue aumentando la población en términos absolutos y cientos de hectáreas se agregan cada año a la mancha urbana. Esto revela cuán equivocada es la tesis del crecimiento (urbano) sostenido como solución a los problemas sociales. Ya que a mayor crecimiento poblacional y de la plancha urbana, mayores son los índices de degradación ambiental. De ahí que siempre se debe tener en mente la necesidad de frenar y neutralizar tal avance, mediante el reordenamiento del ecosistema urbano en función de los ecosistemas naturales y humano.

De lo señalado se desprende un elemento enriquecedor (y un freno de la urbanización salvaje) para el análisis y perspectivas de las grandes ciudades (como la zмvм, Guadalajara y Monterrey), y éste es el papel de la producción regional y local agropecuaria, agroalimentaria, específicamente. Esto es sólo un aspecto de la revaloración de lo rural y de los ecosistemas naturales, para garantizar que el futuro inmediato de la humanidad en este planeta asegure el espacio de reproducción de Gaia (Lovelock, 2007). Ello desde luego dentro del proceso de construcción de la nueva urbanidad (y civilidad).

Lo anterior resulta esencial para la sociedad mexicana, ya que el suministro de alimentos no puede dejarse simplemente a la importación desde otras regiones (menos de vender nuestros recursos naturales estratégicos), ya que primero hay que atender la producción local, dada nuestra estructura productiva y social, ${ }^{9}$ aparte del hecho de que su producción local implica una precondición energética esencial (energía calorífica) para

${ }^{9}$ Esto es, vislumbrando el efecto global y no verlo como una ecuación aislada. 
la reposición de la energía humana y, con ella, las demás transformaciones energéticas realizadas por el hombre; de lo que resulta también una necesaria reducción en el consumo de energía, con el consecuente ahorro de dinero y de recursos naturales.

El desaprovechamiento de los recursos agrícolas de la ciudad-región no debe entenderse como algo que está ocurriendo inevitablemente, ya que se trata de un hecho que debiera corregirse (mediante el impulso de los mercados agropecuarios, agroindustriales y alimentarios), pues la sustentabilidad alimentaria es parte de la sustentabilidad en general, y urbana en particular. Esto, por supuesto, se relaciona con los programas de agricultura urbana (Schenna, 1995) impulsados por la ONU. Por tanto, este aspecto es parte de la solución a la crisis ambiental global-local.

Las consecuencias del modelo de acumulación del capital urbano en la ZMVM han sido desastrosas. El gobierno y la ciudadanía no han estado a la altura; se han movilizado pero no se han dado los resultados que todos esperamos. El problema no sólo es de voluntad política, que ha faltado, sino también de la inconveniencia en querer realmente cambiar el estado de cosas, y de ánimo, de la gran ciudad, a veces amable, casi siempre violenta. Tampoco es suficiente pensar que con una propuesta muy adecuada pueda salirse del atolladero en el ámbito social y ecológico.

En suma, es necesario reconocer que la respuesta apropiada socialmente con un criterio de corresponsabilidad en la evolución natural, humana y frente al desarrollo social (que son los requisitos de la sustentabilidad, específicamente urbana), implica considerar que el problema ambiental está ligado a la construcción de una conciencia socioambiental, la reversión de las tendencias de las instituciones gubernamentales a la depredación y la adecuación de las políticas públicas hacia el cambio de paradigma. Pero sobre todo hay que ubicarse en el contexto de las políticas no gubernamentales, los movimientos ambientalistas de toda índole y la integración de redes sociales, como medios fundamentales para enfrentar la degradación ambiental del ecosistema urbano y avanzar así hacia su reversión.

Por último, el eje de las alternativas urbanas de sustentabilidad se finca en que el nuevo estilo de desarrollo de la ciudad debe comprenderse más allá de la suma mecánica de sus componentes económico, ecológico y social, ya que la interacción entre ellos revela que dicho desarrollo debe ser compatible al menos con: 1) los ecosistemas naturales; 2) las actividades agropecuarias y alimenticias; 3) el fomento a las actividades domésticas, públicas, industriales y de servicios, de manera orgánica (integrada), y en función de una economía de energía, y 4) la conclusión del ciclo de los desechos y su recuperación, así como su disposición final apropiada. El resultado es que con ello se garantizaría la renovación de los ecosistemas en compatibilidad con la economía, la sociedad y, desde 
luego, el territorio. Y todo lo anterior en el marco de una nueva urbanidad (Torres, 2006: 239) y de la más amplia participación ciudadana.

Estos elementos se integran formando una unidad, que con el modelo actual, resulta incompatible con las necesidades socioambientales, ya que constituye un proceso fragmentado espacialmente y con distintas formas de ruptura urbana (y anacronismos históricos, como la mezcla de agua de lluvia con el drenaje y la descarga de aguas negras a los cuerpos superficiales, entre muchas otras).

Finalmente, la lucha contra la degradación ambiental (desde la perspectiva ecosocial) es una condición sine qua non es posible la sustentabilidad urbana o, mejor dicho, un desarrollo compatible entre el campo y la ciudad. Esto significa: la revolución energética, sustentabilidad alimentaria y del agua, nuevas tecnologías y la economía de los desechos. Y todo ello en el proceso de construcción/reconstrucción de las nuevas ciudades.

\subsection{La sustentabilidad de la ZMVM y la planeación territorial}

Construir el desarrollo sustentable para la ZMvM, con las condiciones del desarrollo rural y urbano que la han condicionado, toma cuenta de una necesaria planeación, dentro y entre los distintos territorios; e implica una dimensión planetaria; es decir, no sólo en función del mercado, como una expresión del proceso de producción visto en su conjunto, ya que sin plan (en forma de planeación o planificación) no se podría reducir la depredación socioambiental, ${ }^{10}$ así como avanzar a nuevos procesos civilizatorios en el ámbito planetario.

La sustentabilidad territorial se refiere a cómo coinciden los distintos momentos del ciclo natural con el socioeconómico en un delimitado espacio geográfico; por supuesto, la sustentabilidad se realiza más allá del territorio, por lo que no está limitada por éste, mientras que el ordenamiento territorial sí depende de aquélla.

Se puede afirmar, sin lugar a dudas, que en la práctica el discurso de la sustentabilidad territorial ha representado en la ZMvM una cuestión de alianzas políticas (por ejemplo, entre el Estado de México y el D.F.), entre los distintos municipios y delegaciones que son nodos territorializados del poder económico y político, y que aportan el sustrato humano (esto es, los nuevos súbditos) y natural (nuevas áreas territoriales) para la expansión y profundización de la ciudad de ciudades, como México, y que se reparten dicho botín.

\footnotetext{
${ }^{10}$ A este respecto es interesante el Plan Verde propuesto por el gobierno del D.F. Su principal defecto es que no aplica en la ZMVM sino sólo en el territorio del D. F.
} 
En este contexto resulta evidente que no hay manera de avanzar hacia la planeación territorial si no se reconoce que los problemas de la ZMVM derivan en gran medida de que todavía se abordan de manera separada entre el D.F. y el Estado de México, y no existe una entidad metropolitana como tal, lo que dificulta resolver su problemática socioambiental.

En un análisis teórico, el territorio es un concepto relativo, pues no tiene que ver con limitaciones reales, sino imaginarias socialmente. Por ello, las transformaciones estructurales que requiere la ZMVM se deben basar en un enfoque de la territorialidad (y el mismo concepto de ciudad) que no ignore otros (más amplios) y que supone desde luego el empoderamiento social (empowerment), en que se habilite a la gente para participar activa y coordinadamente (Graizbord, 1999) en el marco comunitario, local, regional, nacional e internacional y bajo el principio de la planetariedad; de lo contrario, la idea de territorio quedaría diluida como una mera expresión del proceso de yuxtaposición de círculos concéntricos urbanísticos, que avanzan irremediablemente a la concentración y consolidación del poder (en sentido amplio) en de un espacio físico específico; y actualmente también, a la concentración del territorio en el poder.

\section{Conclusiones}

En este artículo se reconoce la importancia del enfoque territorial para el análisis e interpretación del entramado urbano o rural (sobre todo a partir de sus múltiples interacciones), pese a las limitaciones estudiadas aquí en el sentido de que es parcial e insuficiente.

Por tanto no debiera desecharse, aunque éste es sólo un elemento de una concepción más amplia que debe abarcar desde lo global-local hasta lo local-global. De esta manera constituye nada más un momento aislado en la cadena de la sustentabilidad y compatibilidad de la vida frente al desarrollo, y desde el punto de vista del ciclo ecosocial. Empero, analizado críticamente, el enfoque podría adecuarse muy bien a un expansionismo territorial megalopolitano (que incluye la desterritorialización/reterritorialización), ya que el territorio se encuentra empalmado con el poder económico y político.

La territorialidad entonces debiera acompañarse de la multisectorialidad, transversalidad y planetariedad, para mejorar así de manera más integral el desarrollo urbano en consonancia con la lucha frente al calentamiento global y la crisis ambiental-humanitaria. Y es que la vida urbana de la megalópolis choca con la integridad de los ecosistemas naturales y humanos, así como con los agroecosistemas (alimentarios). La reversión de este hecho es el principio fundamental de la sustentabilidad urbana. 
Por tanto, el dilema entre megalópolis (zMvM) o la construcción de una red de ciudades verdes ${ }^{11}$ se puede resolver a favor de estas últimas, lo que supone desmontar la depredación urbana mediante el rescate ecosistémico y agroecosistémico de los territorios, lo que implica intercalar áreas verdes entre las construcciones y configurar cordones ecológicos que posibiliten poner a la ciudad al servicio de la conservación y restauración de la naturaleza, así como de las áreas productoras de alimentos desde una perspectiva de equidad social.

Lo anterior implica la construcción de nuevos territorios a partir del reordenamiento ecosistémico y poblacional, avanzando hacia una planeación territorial que reconozca la unidad territorial de la ZMVM para resolver los problemas urbanos con una perspectiva metropolitana. Esto quiere decir que es necesario combatir la fragmentación de los ecosistemas naturales, lo cual implica compactar territorialmente, y descomprimir urbanísticamente a la vez, la zona urbana (reduciendo la explosión poblacional urbana en el contexto nacional y no sólo de la ciudad-región).

Por tanto, el enfoque territorial metropolitano se debe combinar con el planetario, que requiere una visión integral de la naturaleza. Esto quiere decir que sólo así se podrá responder a los retos de una sustentabilidad urbana como expresión espacial del ciclo ecosocial.

Asimismo, es necesario impulsar una red de ecocomunidades con amplia participación social, sin la cual las políticas públicas y la reorientación de la empresa privada se mostrarían incapaces por sí solas para coadyuvar a ensanchar el camino de la sustentabilidad urbana de la ZMVM.

Ante la crisis múltiple y global de las ciudades (alimentaria, de agua, de energía, ambiental y socioeconómica), que en realidad es una crisis de la civilización urbana, es imperiosa la necesidad de enfrentar el previsible shock urbano, ${ }^{12}$ y avanzar en la formulación y realización de propuestas que se traduzcan en acciones (desde los barrios y las comunidades) que posibiliten la reducción de la huella ecológica; impedir que la economía y la sociedad rebasen la capacidad de carga de los ecosistemas; y de que sea posible (Hayward, 2005) un espacio ecológico equitativo para sus habitantes. De otra manera, se le estaría dando continuidad al modelo urbano depredador.

Finalmente, la estrategia local-global del desarrollo territorial es avanzar hacia la construcción de una nueva urbanidad (que surge como base

\footnotetext{
${ }^{11}$ Obviamente esto tiene que ver con la idea de ciudad jardin de Ebenezer Howard en su "Garden cities of tomorrow" (Huang y Shu, 2010).

${ }^{12}$ La situación vivida en la ciudad de México en materia de escasez de agua durante la Semana Santa del año 2009, no es una casualidad sino un ajuste drástico que ejemplifica lo que viene y que acompańa a la expansión territorial de la zona urbana; y ni qué decir del pánico epidemiológico posterior que paralizó a la ciudad (y al país).
} 
de la ciudadanía global), lo que presupone un nuevo trato entre el campo y la ciudad y la planeación del territorio con los presupuestos analizados.

\section{Bibliografía}

Aguilar, Adrián Guillermo (coord.) (2003), Procesos metropolitanos y grandes ciudades, Universidad Nacional Autónoma de México-Miguel Ángel Porrúa-Cámara de Diputados, LIX Legislatura, México.

Barton, Jonathan (2006), "Sustentabilidad urbana como planeación estratégica”, Revista EURE, XXXII (96), Santiago de Chile, pp. 27-45.

Biagini, Hugo y Arturo Roig (2008), Diccionario del pensamiento alternativo, Biblos, Buenos Aires.

Bookchim, Murray (1978), Los límites de la ciudad, Blume, Madrid.

Borja, Jordi y Manuel Castells (1997), Local y global. La gestión de las ciudades en la era de la información, Taurus, México.

Cantú-Chapa, Rubén (2006), "El ambiente sociourbano a fines del siglo $\mathrm{xx}$ ", Primer Foro Internacional: El medio ambiente sociourbano regional. Retos y perspectivas, Instituto Politécnico NacionalCIIEMAD, México.

Castells, Manuel (1983), La cuestión urbana, Siglo XXI, México.

Davis, Mike (2003), Dead cities: and other tales, The New Press, Nueva York.

Dempsey, Nicola, Glen Bramel, Sinead Power y Caroline Brown (2009), The social dimension of sustainable development: Definig urban social sustainability, John Wiley and Sons, New Jersey.

Echeverri-Perico, Rafael y Édgar Moscardi (2005), Construyendo el desarrollo rural sustentable en los territorios de México, IICA, México.

Echeverri-Perico, Rafael (2006), "Desarrollo territorial sustentable, el camino hacia la construcción territorial”, Alasru, 3, México, pp. 199-222. 
Ezcurra, Exequiel, Marisa Mazari, Irene Pisanty y Adrián G. Aguilar (2006), La Cuenca de México. Aspectos ambientales criticos y sustentabilidad, Fondo de Cultura Económica, México.

Ferrajoli, Luigi (1995), Derecho y razón, Trotta, Madrid.

Fuente, Juan de la (coord.) (2006), Lo que todo buen chilango debe saber sobre la Ciudad de México, Gobierno del Distrito Federal, México.

García-Canclini, Néstor (2003), "México 2010: una ciudad que improvisa su globalización”, Alteridades, 13 (26), Universidad Autónoma Metropolitana, Iztapalapa, México, pp. 7-14.

Garza, Gustavo (2003), La urbanización de México en el siglo XX, El Colegio de México, México.

Gottmann, Jean (1961), Megalopolis: the urbanized northeastern seabord of the United States, The Twentieth Century Fund, Nueva York.

Graizbord, Boris (1999), "Planeación urbana, participación ciudadana y cambio social”, Economía, Sociedad y Territorio, II (5), El Colegio Mexiquense, Zinacantepec, pp. 149-161.

Graizbord, Boris (2002), "Elementos para el reordenamiento territorial: uso del suelo y recursos", Estudios Demográficos y Urbanos, 17 (2), El Colegio de México, México, pp. 411-423.

Harvey, David (1977), Urbanismo y desigualdad social, Siglo XXI, Madrid.

Harvey, David (2009), Cosmopolitanism and the geographies of freedom, Columbia University Press, Nueva York.

Hayward, Tim (2005), Global justice and the distribution of natural resources, University of Edinburgh, Edimburgo.

Hernández-Franco, Daniel, Mónica Orozco, José Ángel Camacho Prudente, Hadid Vera Llamas Cruz, César Guadalupe Camacho Ramírez y Victoria Angélica Téllez Romero (2002), Concentración de hogares en condiciones de pobreza en el medio urbano, Sedesol, México. 
Huang, Luxin y Du Shu (2010), The integrated urban ecosystem and urban sustainability, Isocarp Congress, Nairobi.

INEGI (Instituto Nacional de Estadística, Geografía e Informática) (2006), Sistema de Cuentas Nacionales. Producto Bruto por entidad federativa 2000-2006, <http//: www. inegi.gob.mx>, 12 de noviembre de 2010 .

Iracheta, Alfonso (1997), Planeación y desarrollo: una visión al futuro, Plaza y Valdés, México.

Iracheta, Alfonso (2004), "Estado de México, la otra cara de la megaciudad", en Peter Ward, México megaciudad: desarrollo y politica (1970-2002), Porrúa-El Colegio Mexiquense, A.c., México, pp. 491-607.

Jung, Jacques (1972), La ordenación del espacio rural, Instituto de Administración Local, Madrid.

Leff, Enrique (2004), Racionalidad ambiental. La reapropiación social de la naturaleza, Siglo XXI, México.

Linck, Tierry (2006), "La economía y la política en la apropiación de los territorios", Alasru, 3, Universidad Autónoma Chapingo, México, pp. 251-285.

Lipietz, Alan (1979), El capital y su espacio, Siglo XXI, México.

Lovelock, James (2007), La venganza de la tierra, Planeta, Madrid.

Marx, Karl (1985), El capital, vol. 8, Siglo XXI, México.

Mc Donough, William, (2002), Waging pace with inteligence design, <http// www.Macdonoghpartners.com>, 10 de enero 2011.

Moreno-Pérez, Orlando (2008), Desarrollo económico y urbanización en el oriente de la Zona Metropolitana de la Ciudad de México, Porrúa, México.

Morín, Edgar y Anne Briggite Kern (1993), Tierra patria, Nueva visión, Buenos Aires. 
Pérez-Corona, Javier (2008), "Reestructuración y nuevas centralidades en la ZMCM", Tercer Congreso sobre Sustentabilidad y Territorialidad en América Latina, Universidad de Medellín, Colombia, 15 de noviembre.

Ramírez-Miranda, César Adrián (2006), "Crítica al enfoque del desarrollo territorial rural”, Alasru, 3, Universidad Autónoma Chapingo, México, pp. 49-80.

Rees, William y Mathis Wackernagel (1996), Our ecological footprint, New Society Publishers, Brittish Columbia.

Schenna, Shabbir (1995), Urban agriculture: food, jobs and sustainable cities, Programa de Desarrollo de las Naciones Unidas, Nueva York.

Schejtman, Alexander y Julio Berdegué (2003), Desarrollo territorial rural, RMISP, Santiago de Chile.

Torres-Carral, Guillermo (2006), Poscivilización: guerra y ruralidad, Plaza y Valdés-Universidad Autónoma Chapingo, México.

Tudela, Fernando (2008), “Transversalidad y políticas públicas para el desarrollo sustentable", II Coloquio Internacional de Medio Ambiente y Desarrollo, Instituto Politécnico Nacional-ciremad, México, 26 de noviembre.

Ward, Peter M. (1990), México: una megaciudad. Producción y reproducción de un medio ambiente urbano, Consejo Nacional para la Cultura y las Artes-Alianza, México.

Ward, Peter M. (2004), México megaciudad: desarrollo y politica, 19702002, Porrúa-El Colegio Mexiquense, México.

Recibido: 25 de junio de 2009. Reenviado: 17 de agosto de 2010. Aceptado: 23 de agosto de 2010.

Guillermo Torres-Carral. Es doctor en ciencias agrícolas por la Universidad Agrícola de Varsovia, Polonia. Es egresado de la Facultad de Economía de la UNAM (1977). Se desempeña como profesor-investigador de tiempo completo, desde 1979, en el Departamento de Sociología Rural 
de la Universidad Autónoma Chapingo, así como en los programas educativos del doctorado de Ciencias Agrarias, de la maestría y licenciatura en Sociología Rural, y del doctorado en Educación Agrícola Superior. Es miembro, desde 1992, del Sistema Nacional de Investigadores (SNI). Sus líneas de investigación son: economía ecológica, alternativas del minifundio mexicano, nueva ruralidad y desarrollo sustentable en México. Entre sus publicaciones más recientes se encuentran: Introducción a la economía política ecológica, Plaza y Valdés-Universidad Autónoma Chapingo, México (2001); Civilización, ruralidad y ambiente, Plaza y Valdés-Universidad Autónoma Chapingo, México (2003); Poscivilización: guerra y ruralidad, Plaza y Valdés-Universidad Autónoma Chapingo, México (2006); El desarrollo sustentable en México, Plaza y Valdés-Universidad Autónoma Chapingo, México (2009); en coautoría: Agricultura ecológica y reconstrucción social, Universidad Autónoma Chapingo, México (2004); "El pago por servicios ambientales y las comunidades indígenas", Revista Ra Ximhai, 2 (1), México, pp. 187-208 (2006); "La urbanización de la pobreza y un modelo alternativo para la región oriente del Estado de México", Textual, 49, Universidad Autónoma Chapingo, México, pp. 131-154 (2007); "La Ley de Desarrollo Rural Sustentable y el campo mexicano", Revista de Geografía Agricola, 40, Universidad Autónoma Chapingo, pp. 55-72 (2008); reseña crítica: "La crisis ambiental en el laberinto de la sustentabilidad”, Economia, Sociedady Territorio, Ix (31), El Colegio Mexiquense, Zinacantepec, pp. 863-879 (2009). 